\title{
A Cross-Platform Software Framework for Medical Image Processing
}

\author{
Koen Van Leemput and Janne Hämäläinen \\ HUS Helsinki Medical Imaging Center \\ University of Helsinki \\ P.O.B. 340, FIN-00029 HUS, Finland \\ \{koen.vanleemput, janne.hamalainen \}@hus.fi
}

\begin{abstract}
In this paper we present a cross-platform software framework for medical image analysis that we make freely available to the medical imaging community 1 . It allows individual software modules to be loaded on demand, providing ad hoc extensibility and maximal software reusability across multiple application areas. We implemented a number of such software modules, and describe their application in research projects and clinical applications at our hospital.
\end{abstract}

\section{Introduction}

With the ever-increasing quantity and quality of data produced by medical imaging devices, post-processing of acquired images is increasingly finding its way into clinical practice and medical research. While modern radiological workstations offer tools for manipulating and visualizing images in three dimensions, specific clinical applications and research projects in our hospital require tailor-made image analysis software that implements more advanced techniques. Since a considerable amount of functionality is shared by many of these applications, we implemented a highly modular software framework for medical image processing that allows common software modules to be re-used in different projects. The framework provides both an end-user application for medical researchers and clinicians, as well as a rapid development environment for emerging algorithms.

\section{Materials and Methods}

The software is centered around the National Library of Medicine Insight Segmentation and Registration Toolkit (ITK). To make ITK operational in our hospital environment, we developed a number of accompanying tools:

- An ITK image class that describes the position of the image grid with respect to the patient using a fully affine transformation. This allows, among other things, to correctly process and visualize oblique MR scans or CT images acquired with the gantry tilted.

\footnotetext{
${ }^{1}$ Interested readers should contact the first author. 


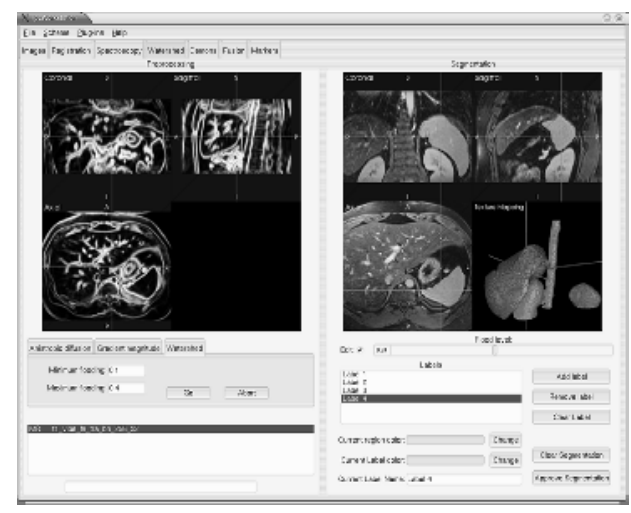

Fig. 1. Specific image analysis tasks can be accomplished by loading a combination of plug-ins that provide the required functionality. This figure shows the plug-in for interactive watershed segmentation.

- An advanced image viewer that can be connected directly to ITK, implemented using the Visualization Toolkit (VTK). It provides arbitrarily oriented multi-planar reconstructions, linked cursors, tracking of curved anatomical structures, as well as 3-D surface and volume rendering.

- ITK classes that use the DICOM Toolkit (DCMTK) to read and write images in the DICOM file format, and to query, retrieve, and send images from and to the hospital PACS system and radiological workstations.

We use the Fast Light Toolkit (FLTK) for the graphical user interface, and provide a mechanism to load so-called plug-ins at run time. Each such a plugin implements specific image processing routines provided by ITK, along with associated user interface and visualization components. The software runs on Windows, Linux and Sun Solaris platforms.

\section{Results and Conclusion}

To date, we have implemented plug-ins for DICOM connectivity, multi-modal registration, deformable registration, interactive watershed segmentation, registration based on internal and external markers, fMRI analysis, and visualization of MEG and EEG source localizations along with structural and functional image data.

The software is currently being used at our hospital in research projects involving morphometry of brain MRI, as well as in treatment planning for boron neutron capture therapy (BNCT). Application and clinical validation in epilepsy surgery planning is scheduled for the forthcoming months. Due to the modular software architecture, future applications will be able to rely on previously implemented plug-ins for common image manipulation tasks, considerably reducing development efforts. 